\title{
Manipulation of Optimal Matchings via Predonation of Endowment*
}

\author{
April 2003 \\ Gloria Fiestras-Janeiroํ, Flip Klijn², Estela Sánchez ${ }^{3}$
}

\begin{abstract}
In this paper we answer a question posed by Sertel and Özkal-Sanver (2002) on the manipulability of optimal matching rules in matching problems with endowments. We characterize the classes of consumption rules under which optimal matching rules can be manipulated via predonation of endowment.
\end{abstract}

Keywords: matching, endowments, manipulation

JEL classification: $\mathrm{C} 78$

\section{Introduction}

In a recent paper, Sertel and Özkal-Sanver (2002), hereafter S \& Ö-S, explained that in two-sided matching models "... the consumption possibilities of an agent may depend on the respective endowments of the pair in which the agent ends up under a matching" (S \&

*We thank Bettina Klaus, Jordi Massó, and İpek Özkal-Sanver for valuable comments and conversations. G. Fiestras-Janeiro received financial support from the Spanish Ministerio de Ciencia y Tecnología and the FEDER through projects PB98-0613-C02-02 and BEC2002-04102-C02-02, and from the Xunta de Galicia through grant PGIDT00PXI20703PN. The work of F. Klijn is partially supported by Research Grant BEC2002-02130 from the Spanish Ministerio de Ciencia y Tecnología and by a Marie Curie Fellowship of the European Community programme "Improving Human Research Potential and the Socioeconomic Knowledge Base" under contract number HPMF-CT-2001-01232. The work of E. Sánchez is supported by project BEC2002-04102-C02-02 from the Spanish Ministerio de Ciencia y Tecnología and the FEDER.

${ }^{1}$ Depart. de Estatística e Investigación Operativa, Universidade de Vigo, Spain; fiestras@uvigo.es

${ }^{2}$ Corresponding author. CODE and Departament d'Economia i d'Història Econòmica, Universitat Autònoma de Barcelona, Edifici B, 08193 Bellaterra, Spain. Tel. (34) 93581 1720; Fax. (34) 935812012 ; fklijn@pareto.uab.es

${ }^{3}$ Depart. de Estatística e Investigación Operativa, Universidade de Vigo, Spain; esanchez@uvigo.es 
Ö-S, p. 66). They endowed the classical marriage matching model (cf. Gale and Shapley (1962)) with two additional features: endowments of some resource and consumption rules which determine how endowments are consumed jointly, i.e., within a matched pair.

In their study of this extended model, $\mathrm{S} \&$ Ö-S focused on the manipulability of the man optimal matching rule $\varphi_{M}$ (for the woman optimal matching rule one simply changes the roles of men and women). ${ }^{4}$ More specifically, they considered four types of manipulation: destruction, hiding, perfect hiding, and predonation of endowments. Mostly under the assumption that there are at least three agents on each side of the market, they characterized the class of consumption rules under which $\varphi_{M}$ is vulnerable to manipulation of any of the first three types. Regarding manipulation by predonation of endowment, they only provided examples of matching markets to show that under several 'natural' consumption rules $\varphi_{M}$ is prone to manipulation. Subsequently, S \& Ö-S (p. 80) posed a research question: Which are the maximal classes of consumption rules under which $\varphi_{M}$ can be manipulated via predonation of endowment?

In this paper we answer this question. We first characterize for any of the four cases of predonation (i.e., man to woman, man to man, woman to man, and woman to woman) the class of consumption rules under which $\varphi_{M}$ is manipulable. We use conditions that are very similar to the ones used by S \& Ö-S, which make a clear comparison with their characterizations possible. Finally, we derive from our characterization results that $\varphi_{M}$ is only non-manipulable under the trivial consumption rule, where each agent consumes exactly his own endowment. (S \& Ö-S already noted that at least under this consumption rule, $\varphi_{M}$ is non-manipulable.) The proofs of our characterizations are considerably different from the proofs in S \& O-S regarding destruction or (perfect) hiding of endowment. This is a consequence of the fact that predonation of endowment typically affects the preferences of many more agents than destruction or (perfect) hiding of endowment.

Predonation of endowment can be related to the concept of bribing on which only very recently some work has been done. Schummer (2000) studied bribe-proof rules in a very broad class of economies. He showed that if the domain of preferences is sufficiently rich then any bribe-proof rule is a constant function. Eső and Schummer (2003) studied the equilibria of a game in the context of a two-bidder, second-price auction where one bidder may bribe the other to commit to stay away from the auction. Massó and Neme (2003) characterized bribe-proof rules in the context of a division problem with one divisible good and single-peaked preferences. The two main differences between these papers and ours are the following. In the first place, a bribe is not shared with other agents, while in our model a predonation is typically also allocated to a third agent according to a consumption rule. In the second place, a bribe only leads to a change of the type of the receiving agent, whereas in our model a predonation may affect other agents' preferences as well.

The paper is organized as follows. In Section 2, we recall the model and notation. Moreover, we recall two results on the manipulability of optimal matching rules in the marriage model, which facilitate the exposition of our proofs. In Section 3, we provide

\footnotetext{
${ }^{4}$ For a comprehensive account on manipulation in the marriage model we refer to Roth and Sotomayor (1990), and for a short review on manipulation of endowments in other contexts we refer to S \& Ö-S.
} 
our characterization results. Finally, in Section 4, we summarize our results and conclude with some examples and remarks.

\section{The model}

The model and most of the notation presented next are due to S \& Ö-S. We consider two finite and disjoint sets $M=\left\{m_{1}, \ldots, m_{r}\right\}$ of men and $W=\left\{w_{1}, \ldots, w_{s}\right\}$ of women where possibly $r \neq s$. Since S \& Ö-S need the assumption $r, s \geq 3$ for most of their results we make this assumption from the start. The set $A=M \cup W$ is called a society. For each agent $i \in A$, we define the potential set of mates as

$$
A(i):= \begin{cases}W \cup\{i\} & \text { if } i \in M \\ M \cup\{i\} & \text { if } i \in W .\end{cases}
$$

Let $\mathcal{R}_{+}=\{x \in \mathcal{R}: x \geq 0\}$. For each $i \in A$, let $v_{i}: A(i) \rightarrow \mathcal{R}_{+}$be a map that totally orders $A(i)$ meaning that for each $i \in A, v_{i}(j)=v_{i}(k)$ if and only if $j=k \in A(i)$. A valuation profile is a set of maps $v=\left\{v_{i}\right\}_{i \in A}$.

We will assume that each agent $i \in A$ has an initial endowment $e_{i} \in \mathcal{R}_{+}$. A vector $e=\left(e_{i}\right)_{i \in A} \in E:=\mathcal{R}_{+}^{A}$ is called an endowment profile. Endowments are allocated between matched agents according to some exogenous consumption rule, which for agent $i \in A$ is a function $c_{i}: A(i) \times E \rightarrow \mathcal{R}_{+}$. For any $j \in A(i)$ and any $e \in E, c_{i}(j, e)$ denotes the consumption of $i$ when matched to $j$. We require that the functions $\left\{c_{i}\right\}_{i \in A}$ satisfy the following three conditions. First, we require that no matter who $i$ 's mate is, he/she does not consume more than their total endowment, i.e.,

$$
C_{1}: c_{i}(j, e) \leq e_{i}+e_{j} \text { for all } j \in A(i) \backslash\{i\} \text { and } e \in E
$$

Second, every self-matched agent consumes his own endowment, i.e.,

$$
C_{2}: c_{i}(i, e)=e_{i} \text { for all } e \in E
$$

The third condition is a combined monotonicity and independency property:

$$
C_{3}: c_{i}(j, e) \leq c_{i}\left(j, e^{\prime}\right) \text { for all } j \in A(i) \text { and } e, e^{\prime} \in E \text { with } e_{i} \leq e_{i}^{\prime} \text { and } e_{j} \leq e_{j}^{\prime} \text {. }
$$

Note that for $i \in A, j \in A(i) \backslash\{i\}$ we do not demand feasibility in the sense that $c_{i}(j, e)+$ $c_{j}(i, e) \leq e_{i}+e_{j}$.

Lemma 1 For all $i \in A$ and $j \in A(i) \backslash\{i\}$ there exists a non-decreasing function $\gamma^{i j}$ : $\mathcal{R}_{+} \times \mathcal{R}_{+} \rightarrow \mathcal{R}_{+}$such that $\gamma^{i j}(0,0)=0$ and $\gamma^{i j}\left(e_{i}, e_{j}\right)=c_{i}(j, e)$ for any $e \in E$. 
Proof. It is straightforward from $C_{1}$ and $C_{3}$.

In view of Lemma 1 and condition $C_{2}$ we conveniently define for any $i \in A$ a function $\gamma^{i i}: \mathcal{R}_{+} \times \mathcal{R}_{+} \rightarrow \mathcal{R}_{+}$as $\gamma^{i i}\left(e_{i}, e_{i}\right):=c_{i}(i, e)=e_{i}$ for any $e \in E$. For each agent $i \in A$, we identify the consumption rule $c_{i}$ with the associated set of functions $\gamma=\left\{\gamma^{i j}\right\}_{j \in A(i)}$. We denote by $\Gamma$ the collection of these associated set of functions $\gamma$.

$\mathrm{S} \&$ Ö-S provided the following examples of consumption rules, which will be discussed later,

1. Trivial: $\gamma_{t}^{i j}\left(e_{i}, e_{j}\right)=e_{i}$ for all $e \in E, i \in A$, and $j \in A(i)$.

2. Reciprocal: $\gamma_{r}^{i j}\left(e_{i}, e_{j}\right)=e_{j}$ for all $e \in E, i \in A$, and $j \in A(i)$.

3. Equal share: $\gamma_{e}^{i j}\left(e_{i}, e_{j}\right)=\frac{1}{2}\left(e_{i}+e_{j}\right)$ for all $e \in E, i \in A$, and $j \in A(i)$.

4. Maximal: $\gamma_{\max }^{i j}\left(e_{i}, e_{j}\right)=\max \left\{e_{i}, e_{j}\right\}$ for all $e \in E, i \in A$, and $j \in A(i)$.

5. Minimal: $\gamma_{\min }^{i j}\left(e_{i}, e_{j}\right)=\min \left\{e_{i}, e_{j}\right\}$ for all $e \in E, i \in A$, and $j \in A(i)$.

6. Public: $\gamma_{p}^{i i}\left(e_{i}, e_{i}\right)=e_{i}, \gamma_{p}^{i j}\left(e_{i}, e_{j}\right)=e_{i}+e_{j}$ for all $e \in E, i \in A$, and $j \in A(i) \backslash\{i\}$.

A matching problem is given by a quadruple $\alpha=(A, e, v, \gamma)$ where $A$ is a society, $e$ is an endowment profile, $v$ is a valuation profile, and $\gamma$ a consumption rule. Note that by taking $e_{i}=0$ for every $i \in A$ we obtain a classical marriage problem (cf. Gale and Shapley (1962)).

Given a matching problem $\alpha=(A, e, v, \gamma)$, each agent $i \in A$ consumes a pair $\left(j, \gamma^{i j}\left(e_{i}, e_{j}\right)\right)$ which consists of a mate $j \in A(i)$ and some amount $\gamma^{i j}\left(e_{i}, e_{j}\right) \in \mathcal{R}_{+}$. The sum $v_{i}(j)+$ $\gamma^{i j}\left(e_{i}, e_{j}\right)$ represents $i$ 's quasi-linear utility if he were matched to agent $j \in A(i)$. For each agent $i \in A$, we define a strict rank order, $R_{i}$, on the set $A(i) \times \mathcal{R}_{+}$as follows. Let $j, k \in A(i)$, then $\left(j, \gamma^{i j}\left(e_{i}, e_{j}\right)\right) R_{i}\left(k, \gamma^{i k}\left(e_{i}, e_{k}\right)\right)$ if

$$
\begin{gathered}
v_{i}(j)+\gamma^{i j}\left(e_{i}, e_{j}\right)>v_{i}(k)+\gamma^{i k}\left(e_{i}, e_{k}\right), \quad \text { or } \\
v_{i}(j)+\gamma^{i j}\left(e_{i}, e_{j}\right)=v_{i}(k)+\gamma^{i k}\left(e_{i}, e_{k}\right) \text { and } \quad v_{i}(j)>v_{i}(k) .
\end{gathered}
$$

With a slight abuse of notation we sometimes write $j R_{i} k$. Furthermore, for all $i \in A, P_{i}$ denotes the strict relation of $R_{i}$ (we need the notation $R_{i}$ because we will be considering situations where $j R_{i} k$ and $j=k$ ).

It is clear that every matching problem $\alpha=(A, e, v, \gamma)$ induces a matching market $(M, W, P)$ (cf. Gale and Shapley (1962)) where $P$ is the profile of strict rank orders as derived above. Let $\alpha=(A, e, v, \gamma)$ be a matching problem. An outcome or matching for $\alpha$ is a bijection $\mu: A \rightarrow A$ such that for all $i \in A, \mu(i) \in A(i)$ and for all $i, j \in A$, $\mu(i)=j$ implies that $\mu(j)=i$. Given $i \in A$ and a matching $\mu, \mu(i)$ is called the mate of agent $i$ under matching $\mu$. The set of all matchings for $A$ is denoted by $\mathcal{M}^{A}$. We say that a matching $\mu \in \mathcal{M}^{A}$ is individually rational for $\alpha$ if and only if for all $i \in A$ we have that $\mu(i) R_{i} i$. A pair of agents $(i, j)$ blocks a matching $\mu \in \mathcal{M}^{A}$ under $\alpha$ if $j P_{i} \mu(i)$ and 
$i P_{j} \mu(j)$. A matching $\mu \in \mathcal{M}^{A}$ is stable for $\alpha$ if and only if it is individually rational for $\alpha$ and there is no blocking pair $(i, j)$ for $\mu$ under $\alpha$.

Gale and Shapley (1962) showed the existence of a stable matching for every matching market. Moreover, they proved that there is a stable matching, $\mu_{M}$, in which every man (woman) gets his best (her worst) mate under stable matchings, and there is a, generally different, stable matching, $\mu_{W}$, in which every man (woman) gets his worst (her best) optimal outcome under stable matchings. Both can be obtained by the so-called deferred acceptance algorithm (cf. Gale and Shapley (1962)).

A matching rule $\varphi$ is a map that associates with each matching problem $\alpha=(A, e, v, \gamma)$ a matching $\varphi[\alpha]=\mu \in \mathcal{M}^{A}$. We define the man optimal matching rule $\varphi_{M}$ as the matching rule that associates with each matching problem $\alpha=(A, e, v, \gamma)$ the man optimal matching $\mu_{M}$ of the corresponding market, i.e., $\varphi_{M}[\alpha]=\mu_{M}$. With the obvious change of roles we define the woman optimal matching rule $\varphi_{W}$.

We use the following two results to establish our characterizations. Theorem 2 tells us that the man optimal matching mechanism in a matching market makes it a (weakly) dominant strategy for each man to state his true preferences. Theorem 3 points out what happens to the optimal matchings in a matching market if some men extend their list of acceptable women. (Clearly, a similar result to Theorem 3 can be obtained by switching the roles of men and women.)

Theorem 2 (Dubins and Freedman (1981); Roth (1982); cf. Theorem 4.7 in Roth and Sotomayor (1990)) Let $(M, W, P)$ be a matching market. Let $P^{\prime}$ be a profile of rank orders ${ }^{5}$ such that for some man $m^{*} \in M$ it holds that $P^{\prime}{ }_{i}=P_{i}, i \neq m^{*}$. Let $\mu_{M}$ and $\mu^{\prime}{ }_{M}$ be the man optimal matchings with respect to $P$ and $P^{\prime}$, respectively. Then, $\mu_{M}\left(m^{*}\right) R_{m^{*}} \mu_{M}^{\prime}\left(m^{*}\right)$.

Theorem 3 (Gale and Sotomayor (1985); cf. Theorem 2.24 in Roth and Sotomayor (1990)) Let $(M, W, P)$ be a matching market with strict rank orders $P$. Let $M^{\prime} \subseteq M$. Let $P^{\prime}$ be a profile of rank orders with $P^{\prime}{ }_{i}=P_{i}$ for all $i \in W \cup\left(M \backslash M^{\prime}\right)$ and where for any $m^{\prime} \in M^{\prime}, P^{\prime} m^{\prime}$ is a strict rank order obtained by adding women to the end of the list of acceptable women in $P_{m}$. Let $\mu_{M}$ and $\mu^{\prime}{ }_{M}\left(\mu_{W}\right.$ and $\left.\mu_{W}^{\prime}\right)$ be the man (woman) optimal matchings with respect to $P$ and $P^{\prime}$, respectively. Then,

$$
\begin{aligned}
& \mu_{M}(m) R_{m} \mu_{M}^{\prime}(m) \text { and } \mu_{W}(m) R_{m} \mu_{W}^{\prime}(m) \text { for all } m \in M, \text { and } \\
& \mu_{M}^{\prime}(w) R_{w} \mu_{M}(w) \text { and } \mu_{W}^{\prime}(w) R_{w} \mu_{W}(w) \text { for all } w \in W .
\end{aligned}
$$

\section{Manipulation via predonation}

Throughout this section we consider a fixed set of agents $A$. Moreover, we assume that any consumption rule satisfies conditions $C_{1}, C_{2}$, and $C_{3}$. Finally, we focus on the man optimal matching rule $\varphi_{M}$, as symmetric results for the woman optimal matching rule $\varphi_{W}$ can easily be obtained by changing the roles of men and women.

\footnotetext{
${ }^{5}$ Rank orders need not be strict for this result.
} 
Definition 4 (Sertel and Özkal-Sanver (2002)) A matching rule $\varphi$ is manipulable via predonation by some agent $i$ to some other agent $j \in A \backslash\{i\}$ if and only if there exist two problems $\alpha=(A, e, v, \gamma)$ and $\alpha^{\prime}=\left(A, e^{\prime}, v, \gamma\right)$ where $e_{i}^{\prime}<e_{i}, e_{j}^{\prime}=e_{j}+e_{i}-e_{i}^{\prime}$ and $e_{r}^{\prime}=e_{r}$ for all $r \in A \backslash\{i, j\}$ such that

$$
\left(i_{\alpha^{\prime}}, \gamma^{i i_{\alpha^{\prime}}}\left(e_{i}^{\prime}, e_{i_{\alpha^{\prime}}}^{\prime}\right)\right) P_{i}\left(i_{\alpha}, \gamma^{i i_{\alpha}}\left(e_{i}, e_{i_{\alpha}}\right)\right)
$$

and

$$
\left(j_{\alpha^{\prime}}, \gamma^{j j_{\alpha^{\prime}}}\left(e_{j}^{\prime}, e_{j_{\alpha^{\prime}}}^{\prime}\right)\right) R_{j}\left(j_{\alpha}, \gamma^{j j_{\alpha}}\left(e_{j}, e_{j_{\alpha}}\right)\right)
$$

where $i_{\alpha}=\varphi[\alpha](i), j_{\alpha}=\varphi[\alpha](j), i_{\alpha^{\prime}}=\varphi\left[\alpha^{\prime}\right](i)$, and $j_{\alpha^{\prime}}=\varphi\left[\alpha^{\prime}\right](j)$.

In order to characterize the class of consumption rules under which the man optimal matching rule can be manipulated via predonation of endowment we recall ${ }^{6}$ from $\mathrm{S} \&$ Ö-S the following two slightly technical, but very weak conditions on consumption rules. For an interpretation of the conditions consider an agent $i$. For expositional convenience we assume that $i=m \in M$. The first condition, Relevancy of Endowment $(\operatorname{RE}(m)$ for short), says that $m$ 's endowment matters to some woman, while keeping her endowment constant. The second condition, Reflexive Relevancy of Endowment $(\operatorname{RRE}(m)$ for short), says that $m$ 's endowment matters to himself, (a) when comparing between the women, or (b) when comparing between the women on the one side and $m$ being single on the other side.

Formally, a consumption rule $\gamma$ satisfies for some $i \in A$ condition

$\mathbf{R E}(i)$ if there exist $j \in A(i) \backslash\{i\}$ and $x, y, z \in \mathcal{R}_{+}$such that

$$
\gamma^{j i}(x, y) \neq \gamma^{j i}(x, z) \text {. }
$$

$\boldsymbol{\operatorname { R R E }}(i, \sim)$ if (a) there exist $j, j^{\prime} \in A(i) \backslash\{i\}, j \neq j^{\prime}$, and $x, y, z, s \in \mathcal{R}_{+}$such that ${ }^{7}$

$$
\gamma^{i j}(z, x)-\gamma^{i j}(y, x) \neq \gamma^{i j^{\prime}}(z, s)-\gamma^{i j^{\prime}}(y, s)
$$

or (b) there exist $j \in A(i) \backslash\{i\}$ and $x, y, z \in \mathcal{R}_{+}$with $y<z$ such that

$$
\gamma^{i j}(z, x)-\gamma^{i j}(y, x) \sim z-y
$$

The symbol $\sim$ in the last condition will stand for $>,<$, or $\neq$, depending on the type of predonation, i.e., man to woman, man to man, etc. This turns out not only to be a concise and convenient way to adapt conditions that S \& Ö-S used in their characterization results, but it also facilitates a comparison of our results with theirs. For instance, they showed that the man optimal matching rule $\varphi_{M}$ is prone to manipulability by a woman

\footnotetext{
${ }^{6}$ More precisely, the only condition from $\mathrm{S} \&$ Ö-S that we generalize is $\operatorname{RRE}(i,>)(b)$ by replacing $>$ with $\sim$ which will stand for $>,<$, or $\neq$. In $\mathrm{S} \&$ Ö-S conditions $\mathrm{RE}$ and RRE were introduced without further interpretation and called $\overline{C_{3}}$ and $C_{3}^{*}$, respectively.

${ }^{7}$ For convenience, we will assume $\gamma^{i j}(z, x)-\gamma^{i j}(y, x)>\gamma^{i j^{\prime}}(z, s)-\gamma^{i j^{\prime}}(y, s)$ and $y<z$.
} 
hiding (or destroying) her endowment if and only if $\gamma$ satisfies $\operatorname{RE}(w)$ or $\operatorname{RRE}(w,>)$ for some $w \in W$.

$\mathrm{S} \&$ Ö-S showed that under consumption rules $\gamma_{r}, \gamma_{e}, \gamma_{\max }, \gamma_{\min }$, and $\gamma_{p}$ the man optimal matching rule $\varphi_{M}$ is manipulable by predonation of endowment from a man to a woman, a man to another man, a woman to a man, and a woman to another woman. As we will see, this is a consequence of the fact that each of these consumption rules satisfies $\operatorname{RE}(i)$ for each $i \in A$ (as can be verified easily).

Next we will characterize for any of the four cases of predonation the class of consumption rules under which $\varphi_{M}$ is manipulable.

\subsection{Predonation from man to woman}

Let $\Gamma_{M W}$ be the class of consumption rules $\gamma \in \Gamma$ for which at least one of the conditions $\operatorname{RE}(w)$ or $\operatorname{RRE}(w,>)$ for some $w \in W$ holds.

Proposition $5 \varphi_{M}$ is manipulable by some man via predonation to some woman if and only if $\gamma \in \Gamma_{M W}$.

Proof. We will first prove the 'if'-part by providing examples that show that if one of the conditions is satisfied then $\varphi_{M}$ is manipulable via predonation of endowment by a man to a woman. All our examples can simply be extended to situations with more men and women by choosing preferences appropriately. We elaborate only the first example. Similar procedures for the other examples are left to the reader.

Example $\operatorname{RE}(w)$, i.e., $\gamma \in \Gamma_{M W}$ because there is some $w \in W$ for which $\operatorname{RE}(w)$ holds. Without loss of generality we assume $w=w_{1}$. Then, there is a man, $m_{2} \in M$, say, and $x, y, z \in \mathcal{R}_{+}$with $z>y$, such that

$$
\gamma^{m_{2} w_{1}}(x, z)>\gamma^{m_{2} w_{1}}(x, y)
$$

We now construct two matching problems $\alpha=(A, e, v, \gamma)$ and $\alpha^{\prime}=\left(A, e^{\prime}, v, \gamma\right)$ where $\alpha^{\prime}$ is obtained by means of a predonation from $m_{1}$ to $w_{1}$. Let the endowments $e$ and $e^{\prime}$ be defined by:

$$
\begin{aligned}
& \text { - } e_{m_{2}}=x, e_{w_{1}}=y, e_{m_{1}}=z-y \text {, and } e_{i} \in \mathcal{R}_{+} \text {for all } i \in A \backslash\left\{m_{1}, m_{2}, w_{1}\right\}, \\
& \text { - } e_{m_{1}}^{\prime}=0, e_{w_{1}}^{\prime}=z \text {, and } e_{j}^{\prime}=e_{j} \text { for all } j \in A \backslash\left\{m_{1}, w_{1}\right\} .
\end{aligned}
$$

Choose the valuation profile $v$ such that it satisfies the following conditions: ${ }^{8}$

$$
\begin{aligned}
& v_{m_{1}}\left(w_{1}\right)>\max _{j \in A\left(m_{1}\right) \backslash\left\{w_{1}, w_{2}\right\}}\left\{v_{m_{1}}(j)+\gamma^{m_{1} j}\left(e_{m_{1}}, e_{j}\right)\right\}-\gamma^{m_{1} w_{1}}\left(e_{m_{1}}, e_{w_{1}}\right), \\
& v_{m_{1}}\left(w_{2}\right)>v_{m_{1}}\left(w_{1}\right)+\max \left\{\gamma^{m_{1} w_{1}}\left(e_{m_{1}}, e_{w_{1}}\right), \gamma^{m_{1} w_{1}}\left(e_{m_{1}}^{\prime}, e_{w_{1}}^{\prime}\right)\right\}-\gamma^{m_{1} w_{2}}\left(e_{m_{1}}^{\prime}, e_{w_{2}}^{\prime}\right),
\end{aligned}
$$

${ }^{8}$ To see that such a choice of $v_{m_{1}}$ is possible, note that we can first choose any value for $v_{m_{1}}\left(m_{1}\right)$ and $v_{m_{1}}\left(w_{r}\right)(r \neq 1,2)$, then $v_{m_{1}}\left(w_{1}\right)$ sufficiently large, and finally $v_{m_{1}}\left(w_{2}\right)$, also sufficiently large. The feasibility of the choice of the other valuations can be seen in a similar way. Note also that we can find $v_{m_{2}}\left(w_{2}\right)$ satisfying (4) and (5) since (1) holds. 


$$
\begin{aligned}
& v_{m_{2}}\left(w_{1}\right)>\max _{j \in A\left(m_{2}\right) \backslash\left\{w_{1}, w_{2}\right\}}\left\{v_{m_{2}}(j)+\gamma^{m_{2} j}\left(e_{m_{2}}, e_{j}\right)\right\}-\gamma^{m_{2} w_{1}}\left(e_{m_{2}}, e_{w_{1}}\right), \\
& \gamma^{m_{2} w_{1}}\left(e_{m_{2}}, e_{w_{1}}\right)-\gamma^{m_{2} w_{2}}\left(e_{m_{2}}, e_{w_{2}}\right)<v_{m_{2}}\left(w_{2}\right)-v_{m_{2}}\left(w_{1}\right), \\
& v_{m_{2}}\left(w_{2}\right)-v_{m_{2}}\left(w_{1}\right)<\gamma^{m_{2} w_{1}}\left(e_{m_{2}}^{\prime}, e_{w_{1}}^{\prime}\right)-\gamma^{m_{2} w_{2}}\left(e_{m_{2}}, e_{w_{2}}\right), \\
& v_{m_{k}}\left(m_{k}\right)>\max _{j \in A\left(m_{k}\right) \backslash\left\{m_{k}\right\}}\left\{v_{m_{k}}(j)+\gamma^{m_{k} j}\left(e_{m_{k}}^{\prime}, e_{j}^{\prime}\right)\right\}-e_{m_{k}}, m_{k} \in M \backslash\left\{m_{1}, m_{2}\right\}, \\
& v_{w_{1}}\left(m_{1}\right)>\max _{j \in A\left(w_{1}\right) \backslash\left\{m_{1}, m_{2}\right\}}\left\{v_{w_{1}}(j)+\gamma^{w_{1} j}\left(e_{w_{1}}^{\prime}, e_{j}^{\prime}\right)\right\}-\gamma^{w_{1} m_{1}}\left(e_{w_{1}}, e_{m_{1}}\right), \\
& v_{w_{1}}\left(m_{2}\right)>v_{w_{1}}\left(m_{1}\right)+\max \left\{\gamma^{w_{1} m_{1}}\left(e_{w_{1}}, e_{m_{1}}\right), \gamma^{w_{1} m_{1}}\left(e_{w_{1}}^{\prime}, e_{m_{1}}^{\prime}\right)\right\}-\gamma^{w_{1} m_{2}}\left(e_{w_{1}}, e_{m_{2}}\right), \\
& v_{w_{2}}\left(m_{1}\right)>\max _{j \in A\left(w_{2}\right) \backslash\left\{m_{1}, m_{2}\right\}}\left\{v_{w_{2}}(j)+\gamma^{w_{2} j}\left(e_{w_{2}}, e_{j}\right)\right\}-\gamma^{w_{2} m_{1}}\left(e_{w_{2}}, e_{m_{1}}^{\prime}\right), \\
& v_{w_{2}}\left(m_{2}\right)>v_{w_{2}}\left(m_{1}\right)+\gamma^{w_{2} m_{1}}\left(e_{w_{2}}, e_{m_{1}}\right)-\gamma^{w_{2} m_{2}}\left(e_{w_{2}}, e_{m_{2}}\right), \\
& v_{w_{k}}\left(w_{k}\right)>\max _{j \in A\left(w_{k}\right) \backslash\left\{w_{k}\right\}}\left\{v_{w_{k}}(j)+\gamma^{w_{k} j}\left(e_{w_{k}}, e_{j}\right)\right\}-e_{w_{k}}, w_{k} \in W \backslash\left\{w_{1}, w_{2}\right\} .
\end{aligned}
$$

Then the first positions in the initial preference system can be concisely depicted as follows:

\begin{tabular}{cccc}
$m_{1}$ & $m_{2}$ & $w_{1}$ & $w_{2}$ \\
\hline$w_{2}$ & $\mathbf{w}_{\mathbf{2}}$ & $m_{2}$ & $m_{2}$ \\
$\uparrow$ & $\mathbf{w}_{1}$ & $\uparrow$ & $m_{1}$ \\
$w_{1}$ & $m_{2}$ & $m_{1}$ & $\uparrow$ \\
$m_{1}$ & & $w_{1}$ & $w_{2}$
\end{tabular}

Here and henceforth we indicate in boldface which positions definitely switch going from $\alpha$ to $\alpha^{\prime}$ due to the choice of $e$ and $v$. The arrows indicate 'sufficient distance' in the valuations so that after a predonation 1) no agent from the upper (lower) part has moved to the lower (upper) part, and 2) the utility levels of the upper part are still higher than the pre-predonation utility levels of the lower part. For instance, the arrow in the first column depicts conditions (2) and (3), which not only guarantee that $w_{2}$ remains agent $m_{1}$ 's favorite mate, but also make sure that the utility from $w_{2}$ after predonation is still higher than the utility from any other agent before the predonation. We have left out any agent not in $\left\{m_{1}, m_{2}, w_{1}, w_{2}\right\}$ because their positions do not influence in the changes of the man optimal matching.

In matching problem $\alpha$, the man optimal stable matching $\mu_{M}^{\alpha}$ is given by

$$
\begin{aligned}
& \mu_{M}^{\alpha}\left(m_{1}\right)=w_{1}, \mu_{M}^{\alpha}\left(m_{2}\right)=w_{2}, \\
& \mu_{M}^{\alpha}\left(m_{k}\right)=m_{k}, \text { for all } m_{k} \in M \backslash\left\{m_{1}, m_{2}\right\}, \text { and } \\
& \mu_{M}^{\alpha}\left(w_{k}\right)=w_{k}, \text { for all } w_{k} \in W \backslash\left\{w_{1}, w_{2}\right\},
\end{aligned}
$$

whereas in $\alpha^{\prime}$, the man optimal stable matching $\mu_{M}^{\alpha^{\prime}}$ is given by

$$
\begin{aligned}
& \mu_{M}^{\alpha^{\prime}}\left(m_{1}\right)=w_{2}, \mu_{M}^{\alpha^{\prime}}\left(m_{2}\right)=w_{1}, \\
& \mu_{M}^{\alpha^{\prime}}\left(m_{k}\right)=m_{k}, \text { for all } m_{k} \in M \backslash\left\{m_{1}, m_{2}\right\}, \text { and } \\
& \mu_{M}^{\alpha^{\prime}}\left(w_{k}\right)=w_{k}, \text { for all } w_{k} \in W \backslash\left\{w_{1}, w_{2}\right\} .
\end{aligned}
$$


From (3), (6), and $C_{3}$ it follows that,

$$
\begin{aligned}
& v_{m_{1}}\left(w_{2}\right)+\gamma^{m_{1} w_{2}}\left(e_{m_{1}}^{\prime}, e_{w_{2}}^{\prime}\right)>v_{m_{1}}\left(w_{1}\right)+\gamma^{m_{1} w_{1}}\left(e_{m_{1}}, e_{w_{1}}\right) \text { and } \\
& v_{w_{1}}\left(m_{2}\right)+\gamma^{w_{1} m_{2}}\left(e_{w_{1}}^{\prime}, e_{m_{2}}^{\prime}\right)>v_{w_{1}}\left(m_{1}\right)+\gamma^{w_{1} m_{1}}\left(e_{w_{1}}, e_{m_{1}}\right) .
\end{aligned}
$$

So the predonation from $m_{1}$ to $w_{1}$ is indeed profitable:

$$
\begin{aligned}
& \left(w_{2}, \gamma^{m_{1} w_{2}}\left(e_{m_{1}}^{\prime}, e_{w_{2}}^{\prime}\right)\right) P_{m_{1}}\left(\left(w_{1}, \gamma^{m_{1} w_{1}}\left(e_{m_{1}}, e_{w_{1}}\right)\right)\right. \text { and } \\
& \left(m_{2}, \gamma^{w_{1} m_{2}}\left(e_{w_{1}}^{\prime}, e_{m_{2}}^{\prime}\right)\right) R_{w_{1}}\left(\left(m_{1}, \gamma^{w_{1} m_{1}}\left(e_{w_{1}}, e_{m_{1}}\right)\right) .\right.
\end{aligned}
$$

The remaining cases $\operatorname{RRE}(w,>)(a)$ and $\operatorname{RRE}(w,>)(b)$ can be analyzed extending the examples below in the same way as we previously have done for $\operatorname{RE}(w)$.

\begin{tabular}{|c|c|c|c|c|}
\hline$m_{1}$ & $m_{2}$ & $m_{3}$ & $w_{1}$ & $w_{2}$ \\
\hline$w_{2}$ & $w_{1}$ & $w_{1}$ & $\mathbf{m}_{3}$ & $m_{2}$ \\
\hline$\uparrow$ & $w_{2}$ & $m_{3}$ & $\mathbf{m}_{2}$ & $m_{1}$ \\
\hline$m_{1}$ & $m_{2}$ & $w_{2}$ & $\uparrow$ & $\uparrow$ \\
\hline \multirow[t]{2}{*}{$w_{1}$} & & & $w_{1}$ & $w_{2}$ \\
\hline & & & $m_{1}$ & $m_{3}$ \\
\hline
\end{tabular}

Examples $\operatorname{RRE}(w,>)(a)$ and $\operatorname{RRE}(w,>)(b)$. Without loss of generality suppose that $w=$ $w_{1}$. Let a man, $m_{1}$, say, predonate to $w_{1}$. Then the following tables show that this is profitable for both $m_{1}$ and $w_{1}$ if we maintain appropriate distances:

\begin{tabular}{cccc}
$m_{1}$ & $m_{2}$ & $w_{1}$ & $w_{2}$ \\
\hline$w_{1}$ & $w_{1}$ & $\mathbf{w}_{1}$ & $m_{2}$ \\
$w_{2}$ & $w_{2}$ & $\mathbf{m}_{2}$ & $m_{1}$ \\
$\uparrow$ & $m_{2}$ & $\uparrow$ & $\uparrow$ \\
$m_{1}$ & & $m_{1}$ & $w_{2}$ \\
\multicolumn{4}{c}{$\operatorname{RRE}\left(w_{1},>\right)(b)$} \\
$j=m_{2}$
\end{tabular}

Now we will prove the 'only if'-part. Let $\gamma \notin \Gamma_{M W}$. Then for any $w \in W$ none of the conditions $\operatorname{RE}(w)$ and $\operatorname{RRE}(w,>)$ holds.

Consider a problem $\alpha=(A, e, v, \gamma)$. Suppose some man predonates some amount of his endowment to some woman. Without loss of generality we assume that $m_{1}$ predonates to $w_{1}$. Let $\alpha^{\prime}=\left(A, e^{\prime}, v, \gamma\right)$ be the resulting problem. We are done if we prove that $m_{1}$ is not strictly better off at $\alpha^{\prime}$.

The predonation causes the following changes in the preferences. It follows from [not $\left.\operatorname{RE}\left(w_{1}\right)\right]$ that $\gamma^{m_{1} w_{1}}(y, x) \leq \gamma^{m_{1} w_{1}}(z, t)$ for all $x, y, z, t \in \mathcal{R}_{+}$with $y<z$ and $y+x=z+t$. Hence,

$$
v_{m_{1}}\left(w_{1}\right)+\gamma^{m_{1} w_{1}}\left(e_{m_{1}}^{\prime}, e_{w_{1}}^{\prime}\right) \leq v_{m_{1}}\left(w_{1}\right)+\gamma^{m_{1} w_{1}}\left(e_{m_{1}}, e_{w_{1}}\right) .
$$

From $C_{2}$ and $C_{3}$ it follows immediately that

$$
v_{m_{1}}(i)+\gamma^{m_{1} i}\left(e_{m_{1}}^{\prime}, e_{i}^{\prime}\right) \leq v_{m_{1}}(i)+\gamma^{m_{1} i}\left(e_{m_{1}}, e_{i}\right) \text { for all } i \in A\left(m_{1}\right) \backslash\left\{w_{1}\right\} .
$$

It also follows from $[\operatorname{not} \operatorname{RE}(w)]$ that

$$
v_{m}(i)+\gamma^{m i}\left(e_{m}^{\prime}, e_{i}^{\prime}\right)=v_{m}(i)+\gamma^{m i}\left(e_{m}, e_{i}\right) \text { for all } m \in M \backslash\left\{m_{1}\right\} \text { and all } i \in A(m) .
$$


From $\left[\operatorname{not} \operatorname{RRE}\left(w_{1},>\right)\right]$ it follows that $z-y \geq \gamma^{w_{1} m}(z, s)-\gamma^{w_{1} m}(y, s) \geq \gamma^{w_{1} m_{1}}(z, t)-$ $\gamma^{w_{1} m_{1}}(y, x)$ for all $m \in M \backslash\left\{m_{1}\right\}$ and $x, y, z, t, s \in \mathcal{R}_{+}$with $y<z$ and $y+x=z+t$. Using these inequalities and again $\left[\operatorname{not} \operatorname{RRE}\left(w_{1},>\right)\right]$ we obtain

$$
\begin{aligned}
\left(v_{w_{1}}\left(w_{1}\right)+e_{w_{1}}^{\prime}\right) & -\left(v_{w_{1}}\left(w_{1}\right)+e_{w_{1}}\right) \geq \\
\left(v_{w_{1}}(\bar{m})+\gamma^{w_{1} \bar{m}}\left(e^{\prime}{ }_{w_{1}}, e^{\prime}{ }_{\bar{m}}\right)\right) & -\left(v_{w_{1}}(\bar{m})+\gamma^{w_{1} \bar{m}}\left(e_{w_{1}}, e_{\bar{m}}\right)\right)= \\
\left(v_{w_{1}}(\tilde{m})+\gamma^{w_{1} \tilde{m}}\left(e^{\prime}{ }_{w_{1}}, e^{\prime}{ }_{\tilde{m}}\right)\right) & -\left(v_{w_{1}}(\tilde{m})+\gamma^{w_{1} \tilde{m}}\left(e_{w_{1}}, e_{\tilde{m}}\right)\right) \geq \\
\left(v_{w_{1}}\left(m_{1}\right)+\gamma^{w_{1} m_{1}}\left(e^{\prime}{ }_{w_{1}}, e_{m_{1}}^{\prime}\right)\right) & -\left(v_{w_{1}}\left(m_{1}\right)+\gamma^{w_{1} m_{1}}\left(e_{w_{1}}, e_{m_{1}}\right)\right) \text { for all } \bar{m}, \tilde{m} \in A\left(w_{1}\right) \backslash\left\{w_{1}, m_{1}\right\} .
\end{aligned}
$$

Condition $C_{3}$ yields

$$
v_{w}\left(m_{1}\right)+\gamma^{w m_{1}}\left(e_{w}^{\prime}, e_{m_{1}}^{\prime}\right) \leq v_{w}\left(m_{1}\right)+\gamma^{w m_{1}}\left(e_{w}, e_{m_{1}}\right) \text { for all } w \in W \backslash\left\{w_{1}\right\} .
$$

Note also that

$$
v_{w}(i)+\gamma^{w i}\left(e^{\prime}{ }_{w}, e^{\prime}{ }_{i}\right)=v_{w}(i)+\gamma^{w i}\left(e_{w}, e_{i}\right) \text { for all } w \in W \backslash\left\{w_{1}\right\} \text { and all } i \in A(w) \backslash\left\{m_{1}\right\}
$$

Next let us turn to an interpretation of inequalities (7)-(12). Inequalities (7) and (8) show us that the utility levels of $m_{1}$ all may drop, but it does not provide any information on whether the positions of mates interchange. Equality (9) shows that the rank order of any other man does not change. From inequality (10) the only possible changes in $w_{1}$ 's rank order are a descent of $m_{1}$ and an ascent of $w_{1}$. Inequalities (11) and (12) show that the only possible change in a woman's $w \neq w_{1}$ rank order is a descent of $m_{1}$.

Let $P_{i}^{0}$ and $P_{i}^{3}$ denote agent $i$ 's rank order in problems $\alpha$ and $\alpha^{\prime}$, respectively. Note that $P_{w_{1}}^{3}$ can be obtained from $P_{w_{1}}^{0}$ in two steps: first we put $m_{1}$ in a (weakly) lower position, and subsequently we put $w_{1}$ in a (weakly) higher position. Let $Q$ be the rank order of agent $w_{1}$ after the first step.

We consider two auxiliary intermediate profiles of rank orders $P^{1}$ and $P^{2}$ for all agents. Define $P_{m_{1}}^{1}:=P_{m_{1}}^{0}$ and $P_{i}^{1}:=P_{i}^{3}$ for $i \in A \backslash\left\{m_{1}, w_{1}\right\}$, and $P_{w_{1}}^{1}:=Q$. Define $P_{m_{1}}^{2}:=P_{m_{1}}^{0}$ and $P_{i}^{2}:=P_{i}^{3}$ for $i \in A \backslash\left\{m_{1}\right\}$. Note that all rank orders are strict; there are no ties. Let $\mu^{0}, \mu^{1}, \mu^{2}$, and $\mu^{3}$ denote the man optimal matchings for rank order profiles $P^{0}, P^{1}, P^{2}$, and $P^{3}$, respectively.

Let $m^{*}:=m_{1}$ and denote $w^{*}:=\mu^{0}\left(m^{*}\right) \in A\left(m^{*}\right)$. In view of inequality (8) we are done if we prove that $w^{*} R_{m^{*}}^{0} \mu^{3}\left(m^{*}\right)$. This will be done in three steps. First we show $w^{*} R_{m^{*}}^{0} \mu^{1}\left(m^{*}\right)$, then $\mu^{1}\left(m^{*}\right) R_{m^{*}}^{0} \mu^{2}\left(m^{*}\right)$, and finally $\mu^{2}\left(m^{*}\right) R_{m^{*}}^{0} \mu^{3}\left(m^{*}\right)$. The result then follows from the transitivity of $R_{m^{*}}^{0}$.

SteP 1: Suppose $\mu^{1}\left(m^{*}\right) P_{m^{*}}^{0} w^{*}$. Then by individual rationality of $\mu^{0}$ at $P^{0}, \tilde{w}:=$ $\mu^{1}\left(m^{*}\right) \in W$ So, $\tilde{w} P_{m^{*}}^{0} w^{*} R_{m^{*}}^{0} m^{*}$ and $\tilde{w} \neq w^{*}$.

Define a new profile of strict rank orders $\bar{P}^{0}$ as follows. Define $\bar{P}_{i}^{0}:=P_{i}^{0}$ for $i \neq m^{*}$, and let $\bar{P}_{m^{*}}^{0}$ be such that $\tilde{w} \bar{P}_{m^{*}}^{0} w^{*} \bar{R}_{m^{*}}^{0} m^{*},\left[w^{*} P_{m^{*}}^{0} m^{*}\right.$ implies $\left.w^{*} \bar{P}_{m^{*}}^{0} m^{*}\right]$, and $m^{*} \bar{P}_{m^{*}}^{0} w$ for all $w \in A\left(m^{*}\right) \backslash\left\{m^{*}, \tilde{w}, w^{*}\right\}$. Similarly, define a profile of rank orders $\bar{P}^{1}$ by $\bar{P}_{i}^{1}:=P_{i}^{1}$ for $i \neq m^{*}$ and $\bar{P}_{m^{*}}^{1}:=\bar{P}_{m^{*}}^{0}$.

Let $\bar{\mu}^{0}$ and $\bar{\mu}^{1}$ denote the man optimal matchings for rank order profiles $\bar{P}^{0}$ and $\bar{P}^{1}$, respectively. Recall that for the man optimal matching mechanism it is always a weakly 
dominant strategy for $m^{*}$ to state his true rank order (Theorem 2). Assume $\bar{\mu}^{0}\left(m^{*}\right) \neq w^{*}$. If $\bar{\mu}^{0}\left(m^{*}\right) \bar{P}_{m^{*}}^{0} w^{*}$ then $\bar{\mu}^{0}\left(m^{*}\right)=\tilde{w}$. Hence, at $P^{0}$ man $m^{*}$ can profit by reporting $\bar{P}_{m^{*}}^{0}$ instead of $P_{m^{*}}^{0}$, a contradiction to truth-telling being weakly dominant. If $w^{*} \bar{P}_{m^{*}}^{0} \bar{\mu}^{0}\left(m^{*}\right)$ then $\bar{\mu}^{0}\left(m^{*}\right)=m^{*}$ by individual rationality of $\bar{\mu}^{0}$ at $\bar{P}^{0}$. Hence, at $\bar{P}^{0}$ man $m^{*}$ can profit by reporting $P_{m^{*}}^{0}$ instead of $\bar{P}_{m^{*}}^{0}$, again a contradiction to truth-telling being weakly dominant. Hence, $\bar{\mu}^{0}\left(m^{*}\right)=w^{*}$. Analogously it follows that $\bar{\mu}^{1}\left(m^{*}\right)=\tilde{w}$.

Since $\tilde{w} \bar{P}_{m *}^{0} w^{*}$ and $\bar{\mu}^{0}$ is the man optimal matching at $\bar{P}^{0}$ it follows that $\bar{\mu}^{1}$ is not stable at $\bar{P}^{0}$.

Suppose $\bar{\mu}^{1}$ is not individual rational at $\bar{P}^{0}$. Then there is an agent $i \neq m^{*}$ such that $i \bar{P}_{i}^{0} \bar{\mu}^{1}(i)$. If $i \in M$ then matching $\bar{\mu}^{1}$ is also not individual rational at $\bar{P}^{1}$ since $\bar{P}_{i}^{0}=\bar{P}_{i}^{1}$, a contradiction. If $i \in W$, then $i \bar{P}_{i}^{0} \bar{\mu}^{1}(i)$ implies $i \bar{P}_{i}^{1} \bar{\mu}^{1}(i)$, and again we find that $\bar{\mu}^{1}$ is also not individual rational at $\bar{P}^{1}$, a contradiction.

Hence, $\bar{\mu}^{1}$ is not stable at $\bar{P}^{0}$ because there exists a blocking pair $(\bar{m}, \bar{w}) \in M \times W$, say. So,

$$
\begin{aligned}
& \bar{m} \bar{P}_{\bar{w}}^{0} \bar{\mu}^{1}(\bar{w}) \quad \text { and } \\
& \bar{w} \bar{P}_{\bar{m}}^{0} \bar{\mu}^{1}(\bar{m}) .
\end{aligned}
$$

On the other hand, $\bar{\mu}^{1}$ is stable at $\bar{P}^{1}$. Hence,

$$
\begin{array}{cc}
\bar{\mu}^{1}(\bar{w}) \bar{P}_{\bar{w}}^{1} \bar{m} & \text { or } \\
\bar{\mu}^{1}(\bar{m}) \bar{P}_{\bar{m}}^{0} \bar{w} . &
\end{array}
$$

(Notice that $\bar{P}_{\bar{m}}^{0}=\bar{P}_{\bar{m}}^{1}$ by construction.) Since (16) contradicts (14) it follows that (15) holds.

If both $\bar{m}, \bar{\mu}^{1}(\bar{w}) \neq m^{*}$, then (15) implies $\bar{\mu}^{1}(\bar{w}) \bar{P}_{\bar{w}}^{0} \bar{m}$, contradicting (13). If $\bar{\mu}^{1}(\bar{w})=m^{*}$ and $\bar{m} \neq m^{*}$, then (13) implies $\bar{m} \bar{P}_{\bar{w}}^{1} m^{*}$, contradicting (15).

Hence, $\bar{m}=m^{*}$. From (14) it follows that $\bar{w} \bar{P}_{m^{*}}^{0} \bar{\mu}^{1}\left(m^{*}\right)$, contradicting that $\bar{\mu}^{1}\left(m^{*}\right)=\tilde{w}$ is $m^{*}$ 's best choice. This completes the first step.

STEP 2: In view of the only difference between the rank orders $P^{1}$ and $P^{2}$ (less men acceptable for $w_{1}$ ), it follows from Theorem 3 (switching the roles of men and women) that $\mu^{1}\left(m^{*}\right) R_{m^{*}}^{1} \mu^{2}\left(m^{*}\right)$. Since $R_{m^{*}}^{1}=R_{m^{*}}^{0}$ we conclude $\mu^{1}\left(m^{*}\right) R_{m^{*}}^{0} \mu^{2}\left(m^{*}\right)$.

STEP 3: It follows from Theorem 2 that $\mu^{2}\left(m^{*}\right) R_{m^{*}}^{2} \mu^{3}\left(m^{*}\right)$. Since $R_{m^{*}}^{2}=R_{m^{*}}^{0}$ it follows that $\mu^{2}\left(m^{*}\right) R_{m^{*}}^{0} \mu^{3}\left(m^{*}\right)$, completing the proof.

\subsection{Predonation from man to man}

Let $\Gamma_{M M}$ be the class of consumption rules $\gamma \in \Gamma$ for which at least one of the conditions $\operatorname{RE}(m)$ or $\operatorname{RRE}(m,<)$ for some $m \in M$ holds.

Proposition $6 \varphi_{M}$ is manipulable by some man via predonation to some other man if and only if $\gamma \in \Gamma_{M M}$. 
Proof. We will first prove the 'if'-part by providing examples that show that if one of the conditions is satisfied then $\varphi_{M}$ is manipulable via predonation of endowment by a man to some other man. All our examples can simply be extended to situations with more men and women by choosing preferences appropriately.

Examples $\operatorname{RE}(m), \operatorname{RRE}(m,<)(a)$, and $\operatorname{RRE}(m,<)(b)$. Suppose without loss of generality $m=m_{2}$. Let $m_{1}$ predonate some endowment to $m_{2}$. Then the following tables show that this is profitable for both $m_{1}$ and $m_{2}$ if we maintain appropriate distances:

\begin{tabular}{cccc}
$m_{1}$ & $m_{2}$ & $w_{1}$ & $w_{2}$ \\
\hline$w_{1}$ & $w_{1}$ & $\mathbf{w}_{\mathbf{1}}$ & $m_{2}$ \\
$w_{2}$ & $\uparrow$ & $\mathbf{m}_{\mathbf{2}}$ & $m_{1}$ \\
$\uparrow$ & $w_{2}$ & $m_{1}$ & $\uparrow$ \\
$m_{1}$ & $m_{2}$ & & $w_{2}$ \\
\hline \multicolumn{4}{c}{$\operatorname{RE}\left(m_{2}\right)$} \\
& $j=w_{1}$
\end{tabular}

\begin{tabular}{cccc}
$m_{1}$ & $m_{2}$ & $w_{1}$ & $w_{2}$ \\
\hline$w_{1}$ & $\mathbf{w}_{1}$ & $m_{2}$ & $m_{2}$ \\
$\uparrow$ & $\mathbf{w}_{2}$ & $m_{1}$ & $m_{1}$ \\
$w_{2}$ & $\uparrow$ & $\uparrow$ & $w_{2}$ \\
$m_{1}$ & $m_{2}$ & $w_{1}$ \\
\hline \multicolumn{4}{c}{$\operatorname{RRE}\left(m_{2},<\right)(a)$} \\
$j=w_{2}, j^{\prime}=w_{1}$
\end{tabular}

\begin{tabular}{ccc}
$m_{1}$ & $m_{2}$ & $w_{1}$ \\
\hline$w_{1}$ & $\mathbf{w}_{\mathbf{1}}$ & $m_{2}$ \\
$\uparrow$ & $\mathbf{m}_{\mathbf{2}}$ & $m_{1}$ \\
$m_{1}$ & & $\uparrow$ \\
& \multicolumn{3}{c}{$w_{1}$} \\
\hline \multicolumn{3}{c}{$\operatorname{RRE}\left(m_{2},<\right)(b)$} \\
\multicolumn{3}{c}{$j=w_{1}$}
\end{tabular}

Now we will prove the 'only if'-part. Let $\gamma \notin \Gamma_{M M}$. Then for any man $m \in M$ none of the conditions $\operatorname{RE}(m)$ and $\operatorname{RRE}(m,<)$ holds.

Consider a problem $\alpha=(A, e, v, \gamma)$. Suppose some man predonates some amount of his endowment to some other man. Without loss of generality we assume that $m_{1}$ predonates to $m_{2}$. Let $\alpha^{\prime}=\left(A, e^{\prime}, v, \gamma\right)$ be the resulting problem. We are done if we prove that $m_{1}$ is not strictly better off at $\alpha^{\prime}$.

The predonation causes the following changes in the preferences. It follows from $C_{2}$ and $C_{3}$ that

$$
v_{m_{1}}(i)+\gamma^{m_{1} i}\left(e_{m_{1}}^{\prime}, e_{i}^{\prime}\right) \leq v_{m_{1}}(i)+\gamma^{m_{1} i}\left(e_{m_{1}}, e_{i}\right) \text { for all } i \in A\left(m_{1}\right)
$$

It follows from $[$ not $\operatorname{RE}(m)]$ that

$$
v_{w}(i)+\gamma^{w i}\left(e^{\prime}{ }_{w}, e_{i}^{\prime}\right)=v_{w}(i)+\gamma^{w i}\left(e_{w}, e_{i}\right) \text { for all } w \in W \text { and all } i \in A(w) .
$$

Note that

$$
v_{m}(i)+\gamma^{m i}\left(e_{m}^{\prime}, e_{i}^{\prime}\right)=v_{m}(i)+\gamma^{m i}\left(e_{m}, e_{i}\right) \text { for all } m \in M \backslash\left\{m_{1}, m_{2}\right\} \text { and all } i \in A(m) \text {. }
$$

It follows from $\left[\operatorname{not} \operatorname{RRE}\left(m_{2},<\right)\right]$ that

$$
\begin{aligned}
\left(v_{m_{2}}(\bar{w})+\gamma^{m_{2} \bar{w}}\left(e_{m_{2}}^{\prime}, e_{\bar{w}}^{\prime}\right)\right) & -\left(v_{m_{2}}(\bar{w})+\gamma^{m_{2} \bar{w}}\left(e_{m_{2}}, e_{\bar{w}}\right)\right)= \\
\left(v_{m_{2}}(\tilde{w})+\gamma^{m_{2} \tilde{w}}\left(e_{m_{2}}^{\prime}, e^{\prime} \tilde{w}\right)\right) & -\left(v_{m_{2}}(\tilde{w})+\gamma^{m_{2} \tilde{w}}\left(e_{m_{2}}, e_{\tilde{w}}\right)\right) \geq \\
\left(v_{m_{2}}\left(m_{2}\right)+e_{m_{2}}^{\prime}\right) & -\left(v_{m_{2}}\left(m_{2}\right)+e_{m_{2}}\right) \text { for all } \bar{w}, \tilde{w} \in A\left(m_{2}\right) \backslash\left\{m_{2}\right\} .
\end{aligned}
$$

Inequality (17) shows that the utility levels of $m_{1}$ all may drop, but it does not provide any information on whether the positions of mates interchange. Equalities (18) and (19) 
say that the rank order of any agent $i \in A \backslash\left\{m_{1}, m_{2}\right\}$ does not change. Inequality (20) establishes that the only possible change in $m_{2}$ 's rank order is a descent of $m_{2}$.

Let $P_{i}^{0}$ and $P_{i}^{2}$ denote agent $i$ 's rank order in problems $\alpha$ and $\alpha^{\prime}$, respectively.

We consider an auxiliary intermediate profile of rank orders $P^{1}$ for all agents. Define $P_{m_{1}}^{1}:=P_{m_{1}}^{0}$ and $P_{i}^{1}:=P_{i}^{2}$ for $i \in A \backslash\left\{m_{1}\right\}$. Note that all rank orders are strict; there are no ties. Let $\mu^{0}, \mu^{1}$, and $\mu^{2}$ denote the man optimal matchings for rank order profiles $P^{0}$, $P^{1}$, and $P^{2}$, respectively.

In view of inequality (17) we are done if we prove that $\mu^{0}\left(m_{1}\right) R_{m_{1}}^{0} \mu^{2}\left(m_{1}\right)$. This will be done in two steps. First we show $\mu^{0}\left(m_{1}\right) R_{m_{1}}^{0} \mu^{1}\left(m_{1}\right)$ and subsequently $\mu^{1}\left(m_{1}\right) R_{m_{1}}^{0} \mu^{2}\left(m_{1}\right)$. The result then follows from the transitivity of $R_{m_{1}}^{0}$.

STEP 1: In view of the only difference between the rank orders $P^{1}$ and $P^{0}$ (more women acceptable in $m_{2}$ 's rank order), it follows from Theorem 3 that $\mu^{0}\left(m_{1}\right) R_{m_{1}}^{0} \mu^{1}\left(m_{1}\right)$.

STEP 2: It follows from Theorem 2 that $\mu^{1}\left(m_{1}\right) R_{m_{1}}^{1} \mu^{2}\left(m_{1}\right)$. Since $R_{m_{1}}^{1}=R_{m_{1}}^{0}$ it holds that $\mu^{1}\left(m_{1}\right) R_{m_{1}}^{0} \mu^{2}\left(m_{1}\right)$. This completes the proof.

\subsection{Predonation from woman to man}

Let $\Gamma_{W M}$ be the class of consumption rules $\gamma \in \Gamma$ for which at least one of the conditions $\operatorname{RE}(i)$ and $\operatorname{RRE}(i,>)$ for some $i \in A$ holds.

Proposition $7 \varphi_{M}$ is manipulable by some woman via predonation to some man if and only if $\gamma \in \Gamma_{W M}$.

Proof. We will first prove the 'if'-part by providing examples that show that if one of the conditions is satisfied then $\varphi_{M}$ is manipulable via predonation of endowment by a woman to a man. All our examples can simply be extended to situations with more men and women by choosing preferences appropriately.

Examples $\operatorname{RE}(m), \operatorname{RE}(w), \operatorname{RRE}(m,>)(a), \operatorname{RRE}(m,>)(b), \operatorname{RRE}(w,>)(a)$, and $\operatorname{RRE}(w,>)$ (b). Suppose without loss of generality that $m=m_{1}$ and $w=w_{1}$. Let $w_{1}$ predonate to $m_{1}$. Then the following tables show that this is profitable for both $w_{1}$ and $m_{1}$ if we maintain appropriate distances:

\begin{tabular}{llll}
$m_{1}$ & $m_{2}$ & $w_{1}$ & $w_{2}$ \\
\hline$w_{2}$ & $w_{2}$ & $m_{2}$ & $\mathbf{m}_{\mathbf{2}}$ \\
$\uparrow$ & $w_{1}$ & $\uparrow$ & $\mathbf{m}_{\mathbf{1}}$ \\
$w_{1}$ & $\uparrow$ & $m_{1}$ & $w_{2}$ \\
$m_{1}$ & $m_{2}$ & $w_{1}$ &
\end{tabular}

\begin{tabular}{ccccc}
$m_{1}$ & $m_{2}$ & $m_{3}$ & $w_{1}$ & $w_{2}$ \\
\hline$m_{1}$ & $\mathbf{w}_{\mathbf{1}}$ & $w_{2}$ & $m_{1}$ & $m_{1}$ \\
$\uparrow$ & $\mathbf{w}_{\mathbf{2}}$ & $w_{1}$ & $m_{3}$ & $m_{2}$ \\
$w_{1}$ & $\uparrow$ & $\uparrow$ & $\uparrow$ & $m_{3}$ \\
$w_{2}$ & $m_{2}$ & $m_{3}$ & $m_{2}$ & $w_{2}$ \\
\multicolumn{5}{c}{$w_{1}$} \\
\hline \multicolumn{5}{c}{$j=m_{2}\left(w_{1}\right)$} \\
\end{tabular}




\begin{tabular}{ccccc}
$m_{1}$ & $m_{2}$ & $w_{1}$ & $w_{2}$ & $w_{3}$ \\
\hline $\mathbf{w}_{3}$ & $w_{2}$ & $m_{2}$ & $m_{1}$ & $m_{1}$ \\
$\mathbf{w}_{2}$ & $w_{1}$ & $\uparrow$ & $m_{2}$ & $w_{3}$ \\
$\uparrow$ & $\uparrow$ & $w_{1}$ & $w_{2}$ & $m_{2}$ \\
$m_{1}$ & $m_{2}$ & $m_{1}$ \\
$w_{1}$ & $w_{3}$ \\
\hline \multicolumn{5}{c}{$\operatorname{RRE}\left(m_{1},>\right)(a)$} \\
$j=w_{2}, j^{\prime}=w_{3}$
\end{tabular}

\begin{tabular}{cccc}
$m_{1}$ & $m_{2}$ & $w_{1}$ & $w_{2}$ \\
\hline $\mathbf{m}_{\mathbf{1}}$ & $w_{2}$ & $m_{1}$ & $m_{1}$ \\
$\mathbf{w}_{\mathbf{2}}$ & $w_{1}$ & $m_{2}$ & $m_{2}$ \\
$\uparrow$ & $\uparrow$ & $\uparrow$ & $w_{2}$ \\
$w_{1}$ & $m_{2}$ & $w_{1}$ \\
\\
\hline \multicolumn{4}{c}{$\operatorname{RRE}\left(m_{1},>\right)(b)$} \\
$j=w_{2}$
\end{tabular}

\begin{tabular}{cccccc}
$m_{1}$ & $m_{2}$ & $m_{3}$ & $w_{1}$ & $w_{2}$ & $w_{3}$ \\
\hline$w_{1}$ & $w_{1}$ & $w_{2}$ & $m_{3}$ & $m_{2}$ & $m_{1}$ \\
$\uparrow$ & $\uparrow$ & $w_{1}$ & $\uparrow$ & $m_{3}$ & $w_{3}$ \\
$w_{3}$ & $w_{2}$ & $\uparrow$ & $\mathbf{m}_{\mathbf{2}}$ & $m_{1}$ & $m_{2}$ \\
$\uparrow$ & $w_{3}$ & $w_{3}$ & $\mathbf{m}_{\mathbf{1}}$ & $w_{2}$ & $m_{3}$ \\
$w_{2}$ & $m_{2}$ & $m_{3}$ & $\uparrow$ \\
$m_{1}$ & \multicolumn{5}{c}{$w_{1}$} \\
\hline \multicolumn{5}{c}{$\operatorname{RRE}\left(w_{1},>\right)(a)$} \\
&
\end{tabular}

\begin{tabular}{ccccc}
$m_{1}$ & $m_{2}$ & $m_{3}$ & $w_{1}$ & $w_{2}$ \\
\hline$m_{1}$ & $w_{1}$ & $w_{2}$ & $m_{3}$ & $m_{2}$ \\
$\uparrow$ & $w_{2}$ & $w_{1}$ & $\uparrow$ & $m_{3}$ \\
$w_{1}$ & $m_{2}$ & $\uparrow$ & $\mathbf{m}_{2}$ & $w_{2}$ \\
$w_{2}$ & & $m_{3}$ & $\mathbf{w}_{\mathbf{1}}$ & $m_{1}$ \\
& \multicolumn{5}{c}{$m_{1}$} \\
\hline \multicolumn{5}{c}{$\operatorname{RRE}\left(w_{1},>\right)(b)$} \\
$j=m_{2}$
\end{tabular}

Now we will prove the 'only if'-part. Let $\gamma \notin \Gamma_{W M}$. Then for any $i \in A$ none of the conditions $\operatorname{RE}(i)$ and $\operatorname{RRE}(i,>)$ holds. From $[$ not $\operatorname{RE}(m)]$ it follows that $\gamma^{w m}(x, y)=$ $\gamma^{w m}(x, z)$ for all $w \in W, m \in M$, and $x, y, z \in \mathcal{R}_{+}$. Hence, for each $w \in W$ and each $m \in M$ there exists a function $f^{w m}: \mathcal{R}_{+} \rightarrow \mathcal{R}_{+}$such that $f^{w m}(x)=\gamma^{w m}(x, z)$ for all $x, z \in \mathcal{R}_{+}$. From $[\operatorname{not} \operatorname{RRE}(w,>)(a)]$ it follows that

$$
f^{w m}(x)-f^{w m}(y)=f^{w m^{\prime}}(x)-f^{w m^{\prime}}(y) \text { for all } w \in W, m, m^{\prime} \in M, \text { and } x, y \in \mathcal{R}_{+} .
$$

Since $f^{w m}(0)=\gamma^{w m}(0,0)=0$ for all $w \in W$ and $m \in M,(21)$ yields

$$
f^{w m}(x)=f^{w m^{\prime}}(x) \text { for all } w \in W, m, m^{\prime} \in M \text {, and } x \in \mathcal{R}_{+} .
$$

So for each $w \in W$ there exists a function $g^{w}: \mathcal{R}_{+} \rightarrow \mathcal{R}_{+}$such that

$$
g^{w}(x)=f^{w m}(x)=\gamma^{w m}(x, y)=\gamma^{w m}(x, 0) \leq x \text { for all } m \in M \text { and } x, y \in \mathcal{R}_{+} .
$$

Similarly, $[\operatorname{not} \operatorname{RE}(w)]$ and $[\operatorname{not} \operatorname{RRE}(m,>)(a)]$ imply that for each $m \in M$ there exists a function $g^{m}: \mathcal{R}_{+} \rightarrow \mathcal{R}_{+}$such that

$$
g^{m}(x)=f^{m w}(x)=\gamma^{m w}(x, y)=\gamma^{m w}(x, 0) \leq x \text { for all } w \in W \text { and } x, y \in \mathcal{R}_{+} .
$$


Consider a problem $\alpha=(A, e, v, \gamma)$. Suppose some woman predonates some amount of her endowment to some man. Without loss of generality we assume that $w_{1}$ predonates to $m_{1}$. Let $\alpha^{\prime}=\left(A, e^{\prime}, v, \gamma\right)$ be the resulting problem. We are done if we prove that $w_{1}$ is not strictly better off at $\alpha^{\prime}$.

Clearly, (22) and (23) imply that the rank order of any agent $i \in A \backslash\left\{w_{1}, m_{1}\right\}$ does not change. From $(22)$ and $[$ not $\operatorname{RRE}(w,>)(b)]$ it follows that the only possible change in $w_{1}$ 's rank order is a descent of herself (i.e., more men are acceptable now). From (23) and [not $\operatorname{RRE}(m,>)(b)]$ it follows that the only possible change in $m_{1}$ 's rank order is an ascent of himself (i.e., less women are acceptable now).

Let $P_{i}^{0}$ and $P_{i}^{2}$ denote agent $i$ 's rank order in the initial and new problem, respectively.

We consider an auxiliary intermediate profile of rank orders $P^{1}$ for all agents. Define $P_{w_{1}}^{1}:=P_{w_{1}}^{0}$ and $P_{i}^{1}:=P_{i}^{2}$ for $i \in A \backslash\left\{w_{1}\right\}$. Note that all rank orders are strict; there are no ties. Let $\mu^{0}, \mu^{1}$, and $\mu^{2}$ denote the man optimal matchings for rank order profiles $P^{0}$, $P^{1}$, and $P^{2}$, respectively.

From (22) it follows that $w_{1}$ 's utility levels all may drop. Hence, we are done if we prove that $\mu^{0}\left(w_{1}\right) R_{w_{1}}^{0} \mu^{2}\left(w_{1}\right)$. This will be done in two steps. First we show $\mu^{0}\left(w_{1}\right) R_{w_{1}}^{0} \mu^{1}\left(w_{1}\right)$ and subsequently $\mu^{1}\left(w_{1}\right) R_{w_{1}}^{0} \mu^{2}\left(w_{1}\right)$. The result then follows from the transitivity of $R_{w_{1}}^{0}$. STEP 1: In view of the only change in the rank orders $P^{0}$ and $P^{1}$ (less women acceptable for $\left.m_{1}\right)$, it follows from Theorem 3 that $\mu^{0}\left(w_{1}\right) R_{w_{1}}^{0} \mu^{1}\left(w_{1}\right)$.

STEP 2: The only difference between the rank orders $P^{1}$ and $P^{2}$ is that more men are acceptable for $w_{1}$. From Theorem 3 (switching the roles of men and women) it follows that $\mu^{1}\left(w_{1}\right) R_{w_{1}}^{1} \mu^{2}\left(w_{1}\right)$. Since $R_{w_{1}}^{1}=R_{w_{1}}^{0}$ we have $\mu^{1}\left(w_{1}\right) R_{w_{1}}^{0} \mu^{2}\left(w_{1}\right)$, completing the proof.

\subsection{Predonation from woman to woman}

Let $\Gamma_{W W}$ be the class of consumption rules $\gamma \in \Gamma$ for which at least one of the conditions $\operatorname{RE}(w)$ or $\operatorname{RRE}(w, \neq)$ for some $w \in W$ holds.

Proposition $8 \varphi_{M}$ is manipulable by some woman via predonation to some other woman if and only if $\gamma \in \Gamma_{W W}$.

Proof. We will first prove the 'if'-part by providing examples that show that if one of the conditions is satisfied then $\varphi_{M}$ is manipulable via predonation of endowment by a woman to some other woman. All our examples can simply be extended to situations with more men and women by choosing preferences appropriately.

Examples $\operatorname{RE}(w), \operatorname{RRE}(w, \neq)(a), \operatorname{RRE}(w,<)(b)$, and $\operatorname{RRE}(w,>)(b)$. Let $w_{1}$ predonate to $w_{2}$. Then the following tables show that this is profitable for both $w_{1}$ and $w_{2}$ if we 
maintain appropriate distances:

\begin{tabular}{cccc}
$m_{1}$ & $m_{2}$ & $w_{1}$ & $w_{2}$ \\
\hline $\mathbf{m}_{1}$ & $w_{2}$ & $m_{1}$ & $m_{1}$ \\
$\mathbf{w}_{2}$ & $w_{1}$ & $m_{2}$ & $\uparrow$ \\
$w_{1}$ & $\uparrow$ & $\uparrow$ & $m_{2}$ \\
& $m_{2}$ & $w_{1}$ & $w_{2}$ \\
\hline \multicolumn{4}{c}{$\operatorname{RE}\left(w_{2}\right)$} \\
& $j=m_{1}$
\end{tabular}

\begin{tabular}{|c|c|c|c|}
\hline$m_{1}$ & $m_{2}$ & $w_{1}$ & $w_{2}$ \\
\hline$w_{2}$ & $w_{2}$ & $m_{1}$ & $\mathbf{m}_{1}$ \\
\hline$w_{1}$ & $w_{1}$ & $\uparrow$ & $\mathbf{m}_{2}$ \\
\hline$\uparrow$ & $m_{2}$ & $m_{2}$ & $\uparrow$ \\
\hline$m_{1}$ & & $w_{1}$ & $w_{2}$ \\
\hline
\end{tabular}

\begin{tabular}{ccc}
$m_{1}$ & $w_{1}$ & $w_{2}$ \\
\hline$w_{2}$ & $m_{1}$ & $\mathbf{m}_{\mathbf{1}}$ \\
$w_{1}$ & $\uparrow$ & $\mathbf{w}_{\mathbf{2}}$ \\
$\uparrow$ & $w_{1}$ & \\
$m_{1}$ & &
\end{tabular}

\begin{tabular}{|c|c|c|c|c|c|}
\hline$m_{1}$ & $m_{2}$ & $m_{3}$ & $w_{1}$ & $w_{2}$ & $w_{3}$ \\
\hline$w_{2}$ & $w_{2}$ & $w_{2}$ & $m_{1}$ & $m_{1}$ & $m_{1}$ \\
\hline$w_{1}$ & $w_{1}$ & $w_{3}$ & $m_{3}$ & $\uparrow$ & $m_{2}$ \\
\hline$w_{3}$ & $w_{3}$ & $w_{1}$ & $\uparrow$ & $m_{2}$ & $m_{3}$ \\
\hline \multirow[t]{2}{*}{$m_{1}$} & $m_{2}$ & $\uparrow$ & $\mathbf{m}_{2}$ & $m_{3}$ & $w_{3}$ \\
\hline & & $m_{3}$ & $\mathrm{w}_{1}$ & $w_{2}$ & \\
\hline
\end{tabular}

Now we will prove the 'only if'-part. Let $\gamma \notin \Gamma_{W W}$. Then for any $w \in W$ none of the conditions $\operatorname{RE}(w)$ or $\operatorname{RRE}(w, \neq)$ holds.

Consider a problem $\alpha=(A, e, v, \gamma)$. Suppose some woman predonates some amount of her endowment to some other woman. Without loss of generality we assume that $w_{1}$ predonates to $w_{2}$. Let $\alpha^{\prime}=\left(A, e^{\prime}, v, \gamma\right)$ be the resulting problem. We are done if we prove that $w_{1}$ is not strictly better off at $\alpha^{\prime}$.

The predonation causes the following changes in the preferences. It follows from [not $\mathrm{RE}(w)]$ that

$$
v_{m}(i)+\gamma^{m i}\left(e_{m}^{\prime}, e_{i}^{\prime}\right)=v_{m}(i)+\gamma^{m i}\left(e_{m}, e_{i}\right) \text { for all } m \in M \text { and all } i \in A(m) .
$$

From $[$ not $\operatorname{RRE}(w, \neq)]$ it follows that

$$
\begin{aligned}
\left(v_{w}(\bar{m})+\gamma^{w \bar{m}}\left(e^{\prime}{ }_{w}, e^{\prime}{ }_{\bar{m}}\right)\right) & -\left(v_{w}(\bar{m})+\gamma^{w \bar{m}}\left(e_{w}, e_{\bar{m}}\right)\right)= \\
\left(v_{w}(\tilde{m})+\gamma^{w \tilde{m}}\left(e^{\prime}{ }_{w}, e^{\prime}{ }_{\tilde{m}}\right)\right) & -\left(v_{w}(\tilde{m})+\gamma^{w \tilde{m}}\left(e_{w}, e_{\tilde{m}}\right)\right)= \\
\left(v_{w}(w)+e^{\prime}{ }_{w}\right) & -\left(v_{w}(w)+e_{w}\right) \text { for all } w \in\left\{w_{1}, w_{2}\right\} \text { and all } \bar{m}, \tilde{m} \in M
\end{aligned}
$$

Finally note that

$$
v_{w}(i)+\gamma^{w i}\left(e^{\prime}{ }_{w}, e^{\prime}{ }_{i}\right)=v_{w}(i)+\gamma^{w i}\left(e_{w}, e_{i}\right) \text { for all } w \in W \backslash\left\{w_{1}, w_{2}\right\} \text { and all } i \in A(w)
$$

Equality (24) shows that the utility levels (in particular the rank orders) of the men do not change. Equality (25) says that although all utility levels of agent $w_{2}$ (agent $w_{1}$ ) 
increase (decrease), her rank order does not change. Finally, equality (26) says that the utility levels (in particular the rank order) of any woman $w \in W \backslash\left\{w_{1}, w_{2}\right\}$ do not change.

Note that the rank orders in the initial and new problem are the same. Hence, the man optimal matching at $\alpha$ and $\alpha^{\prime}$ is also the same. Since all of $w_{1}$ 's utility levels (weakly) drop it follows immediately that $w_{1}$ is not strictly better off at $\alpha^{\prime}$.

\section{Concluding remarks}

We have summarized our main results in Table 1. The result in S \& Ö-S that each of the consumption rules $\gamma_{r}, \gamma_{e}, \gamma_{\max }, \gamma_{\min }$, and $\gamma_{p}$ is manipulable in any of the four cases follows easily from the observation that these consumption rules satisfy $\operatorname{RE}(i)$ for each $i \in A$.

Notice that if a consumption rule allows manipulation by a man via predonation to a woman then it also allows manipulation by a woman via predonation to a man and by a woman via predonation to a woman, and vice versa, i.e., $\Gamma_{M W}=\Gamma_{W M} \cap \Gamma_{W W}$. Switching the roles of men and women in Table 1, we obtain a characterization of the consumption rules that allow for manipulation of the woman optimal matching rule $\varphi_{W}$.

\begin{tabular}{|l|l|}
\hline Type of predonation & Domain of consumption rules under which $\varphi_{M}$ is manipulable \\
\hline man to woman & $\Gamma_{M W}=\{\gamma \in \Gamma: \gamma$ satisfies $\operatorname{RE}(w)$ or $\operatorname{RRE}(w,>)$ for some $w \in W\}$ \\
\hline man to man & $\Gamma_{M M}=\{\gamma \in \Gamma: \gamma$ satisfies $\operatorname{RE}(m)$ or $\operatorname{RRE}(m,<)$ for some $m \in M\}$ \\
\hline woman to man & $\Gamma_{W M}=\{\gamma \in \Gamma: \gamma$ satisfies $\operatorname{RE}(i)$ or $\operatorname{RRE}(i,>)$ for some $i \in A\}$ \\
\hline woman to woman & $\Gamma_{W W}=\{\gamma \in \Gamma: \gamma$ satisfies $\operatorname{RE}(w)$ or $\operatorname{RRE}(w, \neq)$ for some $w \in W\}$ \\
\hline
\end{tabular}

Table 1: Domains of manipulating $\varphi_{M}$ via predonation

In Table 2 we present for each particular type of predonation an example of a consumption rule under which the man optimal matching rule cannot be manipulated.

\begin{tabular}{|l|l|}
\hline Type of predonation & $\varphi_{M}$ not manipulable under consumption rule \\
\hline man to woman & $\gamma^{w m}=\gamma_{p}^{w m}, \gamma^{m w}=\gamma_{t}^{m w}, w \in W, m \in M$ \\
\hline man to man & $\gamma^{w m}=\gamma_{t}^{w m}, \gamma^{m w}=\gamma_{p}^{m w}, w \in W, m \in M$ \\
\hline woman to man & $\gamma^{i j}\left(e_{i}, e_{j}\right)=e_{i} / 2$ for all $i \in A, j \in A(i) \backslash\{i\}$ \\
\hline woman to woman & $\gamma^{w m}=\gamma_{p}^{w m}, \gamma^{m w}=\gamma_{t}^{m w}, w \in W, m \in M$ \\
\hline
\end{tabular}

Table 2: Consumption rules under which $\varphi_{M}$ is not manipulable

S \& Ö-S pointed out that under the trivial consumption rule $\gamma_{t}$ the man (or woman) optimal matching rule is non-manipulable by predonation of any agent (man or woman) to any other agent (man or woman). One may wonder whether it is the unique consumption rule with this property. The next corollary answers this question in the affirmative. 
Corollary 9 The only consumption rule under which $\varphi_{M}$ cannot be manipulated by any agent via predonation to any other agent is the trivial consumption rule, i.e., $\gamma_{t}^{i j}(x, y)=x$ for all $i \in A, j \in A(i)$, and $x, y \in \mathcal{R}_{+}$.

Proof. It remains to prove that there is no other consumption rule with this property. Let $\gamma$ be a consumption rule under which $\varphi_{M}$ is non-manipulable. Then $\gamma \notin \Gamma_{W M} \cup$ $\Gamma_{W W} \cup \Gamma_{M M}$. Since $\gamma \notin \Gamma_{W M}$ it follows from the proof of Proposition 7 that for each $i \in A$ there exists a function $g^{i}: \mathcal{R}_{+} \rightarrow \mathcal{R}_{+}$with

$$
g^{i}(0)=0 \text { and } g^{i}(x)=\gamma^{i j}(x, y) \leq x \text { for all } j \in A(i) \backslash\{i\} \text { and } x, y \in \mathcal{R}_{+} .
$$

Moreover, as $\gamma \notin \Gamma_{W W},[\operatorname{not} \operatorname{RRE}(w, \neq)]$ implies that $g^{w}(x)=x$ for all $w \in W$ and $x \in \mathcal{R}_{+}$. Similarly, as $\gamma \notin \Gamma_{M M},[\operatorname{not} \operatorname{RRE}(m,<)]$ implies that $g^{m}(x)=x$ for all $m \in M$ and $x \in \mathcal{R}_{+}$. Hence, $\gamma=\gamma_{t}$.

\section{References}

Dubins, L.E. and Freedman, D.A. (1981) "Machiavelli and the Gale-Shapley Algorithm," American Mathematical Monthly, 88, 485-494.

Eső, P. and Schummer, J. (2003) "Bribing and Signalling in Second Price Auctions," Working Paper, Northwestern University.

Gale, D. and Shapley, L.S. (1962) "College Admissions and the Stability of Marriage," American Mathematical Monthly, 69, 9-15.

Gale, D. and Sotomayor, M.A.O. (1985) "Some Remarks on the Stable Matching Problem," Discrete Applied Mathematics, 11, 223-232.

Massó, J. and Neme, A. (2003) "Bribe-proof Rules in the Division Problem," UFAE and IAE Working Paper, Universitat Autònoma de Barcelona.

Roth, A.E. (1982) "The Economics of Matching: Stability and Incentives," Mathematics of Operations Research, 7, 617-628.

Roth, A.E. and Sotomayor, M.A.O. (1990) Two-Sided Matching: A Study in GameTheoretic Modeling and Analysis. Econometric Society Monograph Series. New York: Cambridge University Press.

Schummer, J. (2000) "Manipulation through Bribes," Journal of Economic Theory, 91, 180-198.

Sertel, M.R. and Özkal-Sanver, İ. (2002) "Manipulability of the Men- (Women-) Optimal Matching Rule via Endowments," Mathematical Social Sciences, 44, 65-83. 